\title{
Análise dos parâmetros urbanísticos quanto ao aproveitamento solar fotovoltaico em áreas urbanas em processo de adensamento em São Paulo ${ }^{1}$
}

\section{Analysis of urban parameters regarding the use of $\mathrm{PV}$ in urban areas in São Paulo in the process of densification}

\author{
Girotti, Carolina'; Marins, Karin Regina de Castro² \\ 1 Universidade de São Paulo, Av. Prof. Almeida Prado, trav.2 nº. 83, Brasil, \\ cgirotti@usp.br \\ 2 Universidade de São Paulo, karin.marins@usp.br.
}

\begin{abstract}
RESUMO
A geração distribuída é uma modalidade de geração de energia elétrica na qual os consumidores podem gerar sua própria energia elétrica. Esta possibilidade é explorada neste trabalho para o sistema fotovoltaico. Para tanto, há necessidade de avaliação e adequação de parâmetros que orientem a definição, especialmente, da forma urbana. Dessa maneira, o objetivo deste trabalho é avaliar os parâmetros urbanísticos que influenciam a definição da forma urbana e, consequentemente, maximizam a geração de energia fotovoltaica, tomando por estudo de caso, o bairro do Belenzinho, no município de São Paulo, em processo de adensamento e verticalização. A avaliação foi realizada por meio do software Rhinoceros $\AA^{\circledR}$ associado aos plug-ins Grasshopper $\AA^{\circledR}$, Diva $\AA^{\circledR}$ e ao algoritmo genético Galapagos propondo identificar os valores dos parâmetros urbanísticos que maximizam a incidência de radiação solar na cobertura das edificações. O presente trabalho apresenta os resultados parciais de uma pesquisa de mestrado. Os resultados indicam que quando se objetiva otimizar a geração de energia fotovoltaica na cobertura de empreendimentos em lotes concentrados no ambiente urbano, na escala de vizinhança, no contexto da legislação urbanística vigente em São Paulo, valores de coeficiente de aproveitamento 3 e 4 , associados a valores de taxas de ocupação de 0,40 se mostram apropriados.
\end{abstract}

Palavras-chave: Urbanismo Paramétrico, Geração Distribuída de Energia (GD), Sistemas Fotovoltaicos, Morfologia Urbana

\section{ABSTRACT}

Distributed generation is a modality for power generation in which consumers can generate their own electricity. This possibility is explored in this paper for photovoltaics. The possibility of consumers to generate their own electricity through the photovoltaics depends on the evaluation and the adequacy of parameters that guide the definition, above all, of urban

1 GIROTTI, C; MARINS, K.R.C. Modelagem dos parâmetros urbanísticos quanto ao aproveitamento solar fotovoltaico em áreas urbanas concentradas em processo de adensamento em São Paulo. In: II SIMPÓSIO NACIONAL DE GESTÃO E ENGENHARIA URBANA: SINGEURB, 2019, São Paulo. Anais... Porto Alegre: ANTAC, 2019. 
morphology. Therefore, the objective of this research is to evaluate the urban parameters that influence the urban morphology and, consequently, to maximize PV generation, in São Paulo, in the neighborhood of Belenzinho, which is in a process of densification and verticalization. The evaluation was performed with the software Rhinoceros $\AA^{\circ}$ associated with Grasshopper $\AA$ and Diva® plug-ins and Galapagos genetic algorithms. Tools were associated to support the identification of the values of the urban parameters that maximize the incidence of solar radiation in the coverage of the buildings. This paper presents the partial results of a master's research. The paper indicates that, to optimize the PV generation in the rooftops of buildings concentrated in the urban environment, in neighborhood scale, in São Paulo's urban planning legislation context, values of floor area ratio 3 and 4 , associated with values of building coverage ratio of 0.40 , seem to be appropriate.

Keywords: Parametric urban design, Distributed Generation (DG), Photovoltaic Systems, Urban morphology.

\section{INTRODUÇÃO}

O sistema elétrico brasileiro conta com grandes usinas de geração centralizada de energia, formado, predominantemente, por fontes térmicas e hídricas e redes de transmissão e distribuição de energia elétrica. A construção de novas usinas hidrelétricas tem sido dificultada, por motivos como a distância entre as unidades de geração de energia e os centros de consumo e seus impactos socioambientais (ABBUD; TANCREDI, 2010; FEARNSIDE, 1999, 2006). Por tais razões, a geração de energia descentralizada, ou seja, próxima aos consumidores, torna-se atraente (SARRALDE et al., 2015). No Brasil, a principal fonte dos sistemas descentralizados de energia elétrica é solar fotovoltaica (BRASIL, 2012, 2015).

Apesar dos centros urbanos estarem aptos por regulação a gerarem energia elétrica, através da Geração Distribuída, os centros urbanos não foram planejados para gerarem energia solar fotovoltaica, o que prescinde da avaliação e adequação de parâmetros urbanos que orientem a definição, sobretudo, da morfologia urbana (FUTCHER; MILLS, 2013; MARINS; ROMÉRO, 2013; MARTINS et al., 2016; MARTINS; ADOLPE; BASTOS, 2014).

A análise da morfologia urbana para maximizar os níveis de radiação solar, que influenciam nas condições de insolação depende da densidade construída. No presente trabalho, a densidade construída é analisada pelo coeficiente de aproveitamento (C.A.) e pela taxa de ocupação do solo (T.O.). O C.A. define a área construída total, pois é um multiplicador que, aplicado à área do lote e dividido pela área do pavimento-tipo, resulta na quantidade de pavimentos da edificação, conforme indica a equação 1.

\section{Multiplicador (C.A.) $x$ Área do lote = Área total do empreendimento} (1)

A T.O. é a divisão da área de projeção da edificação e a área do lote, conforme indica a equação 2. As regulações urbanísticas definem, a T.O. máxima de um lote e o C.A. mínimos e máximos para cada lote, em função da zona urbana.

\section{Multiplicador (T.O.) $x$ Área do lote $=$ Área projetada do empreendimento}

Dessa forma, o objetivo do trabalho é analisar como a variação da morfologia urbana impacta no potencial de geração de energia fotovoltaica na cobertura das edificações, em escala de unidade de vizinhança, no Município de São Paulo, tomando por estudo de caso o bairro do Belenzinho. O estudo analisa a incidência de irradiação solar horizontal exclusivamente na cobertura das edificações.

\section{METODOLOGIA E EXPLANAÇÃO}

O trabalho utilizou a abordagem quantitativa como metodologia de pesquisa, associando-se estudo de caso e simulação gráfica. 
O Belenzinho foi escolhido por compor área de influência da Estação Belém do Metrô, que integra os "Eixos de Estruturação da Transformação Urbana" (EETU), e portanto, estar em área estratégica em processo de adensamento e verticalização, segundo o Plano Diretor de São Paulo (PREFEITURA DO MUNICÍPIO DE SÃO PAULO, 2014). A simulação gráfica conta com ferramentas de programação paramétrica envolvendo a representação dinâmica da forma urbana associada à simulação de radiação solar no estudo de caso.

O processo simplificado da metodologia adotada é ilustrado pela figura 1.

Figura 1 - Esquema processo metodológico de pesquisa
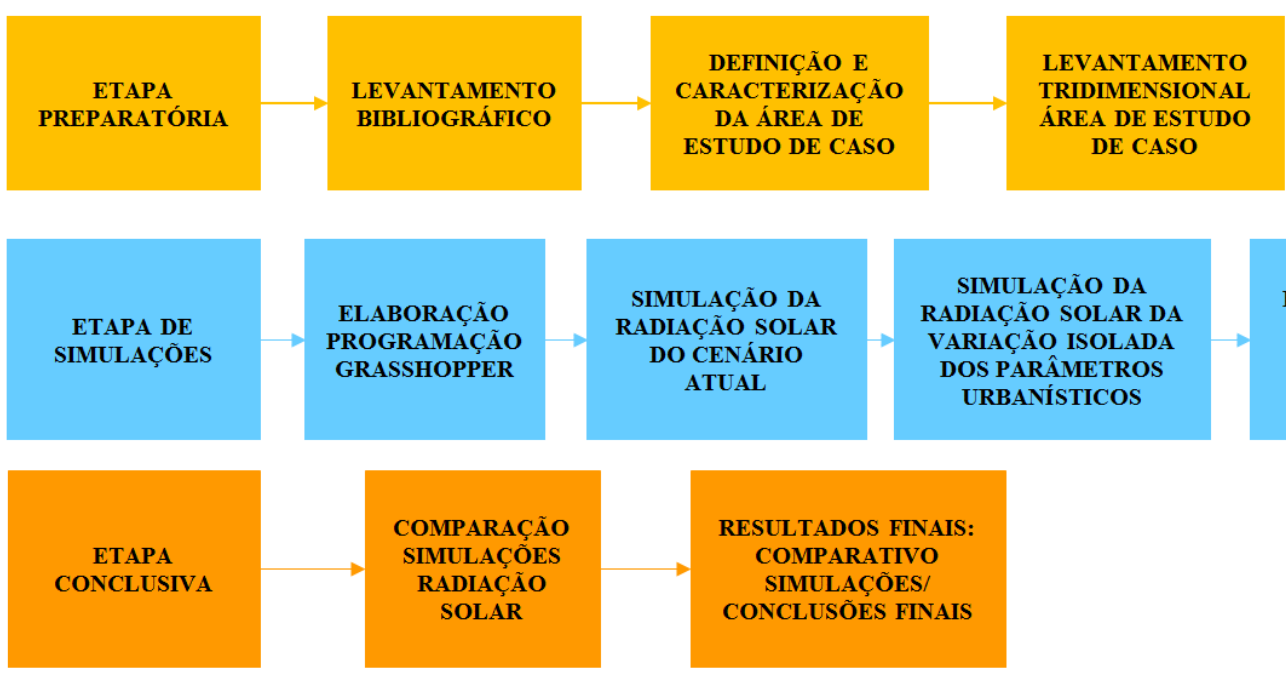

Fonte: As autoras

\subsection{Simulação gráfica: apresentação das ferramentas utilizadas}

A avaliação dos parâmetros urbanísticos foi realizada através do programa de modelagem tridimensional Rhinoceros ${ }^{\circledR}$ (ROBERT MCNEEL \& ASSOCIATES, 2012a), em duas etapas: (1) modelagem da variação isolada dos parâmetros urbanísticos, utilizando o programa Rhinoceros ${ }^{\circledR}$ associado aos plug-ins Grasshopper ${ }^{\circledR}$ (ROBERT MCNEEL \& ASSOCIATES, 2012b) e Diva $B$ (SOLEMMA, 2016), com a finalidade de identificar os valores dos parâmetros urbanísticos que maximizam as respostas da forma urbana em relação à incidência de radiação solar na cobertura das edificações; (2) adicionou-se o algoritmo genético Galapagos, permitindo identificar a melhor combinação dos valores dos parâmetros urbanísticos quanto à maximização da incidência de radiação solar na cobertura das edificações.

O Rhinoceros® foi escolhido por possuir capacidade de interoperabilidade ampliada através de plug-ins, o que o torna flexível com diversos tipos de problemas (ANTON; TǍNASE, 2016), os plug-ins utilizados no trabalho foram:

- $\quad G r a s s h o p p e r \AA$ : principal plug-in do Rhinoceros $\AA$, um editor algorítmico visual que viabiliza a programação com a representação dinâmica dos elementos urbanos (ANTON; TǍNASE, 2016; GAVIRIA; PEREIRA; MIZGIER, 2013; SANTOS; AUER; SOUZA, 2017);

- Diva®: simulador energético que possibilita que a avaliação energética seja incorporada à construção do projeto paramétrico, analisando-se radiação solar difusa e direta (ANTON; TǍNASE, 2016; ROBINSON; STONE, 2004);

- Galapagos: algoritmo genético pertencente ao Grasshopper® que busca soluções que resolvam simultaneamente e satisfatoriamente os diferentes objetivos de um problema através de uma combinação de algoritmos (ANTON; TǍNASE, 2016; MARTINO; CELANI, 2014).

As premissas urbanísticas e climáticas adotadas no trabalho são indicadas no quadro 1. 
Quadro 1 - Premissas gerais adotadas no trabalho

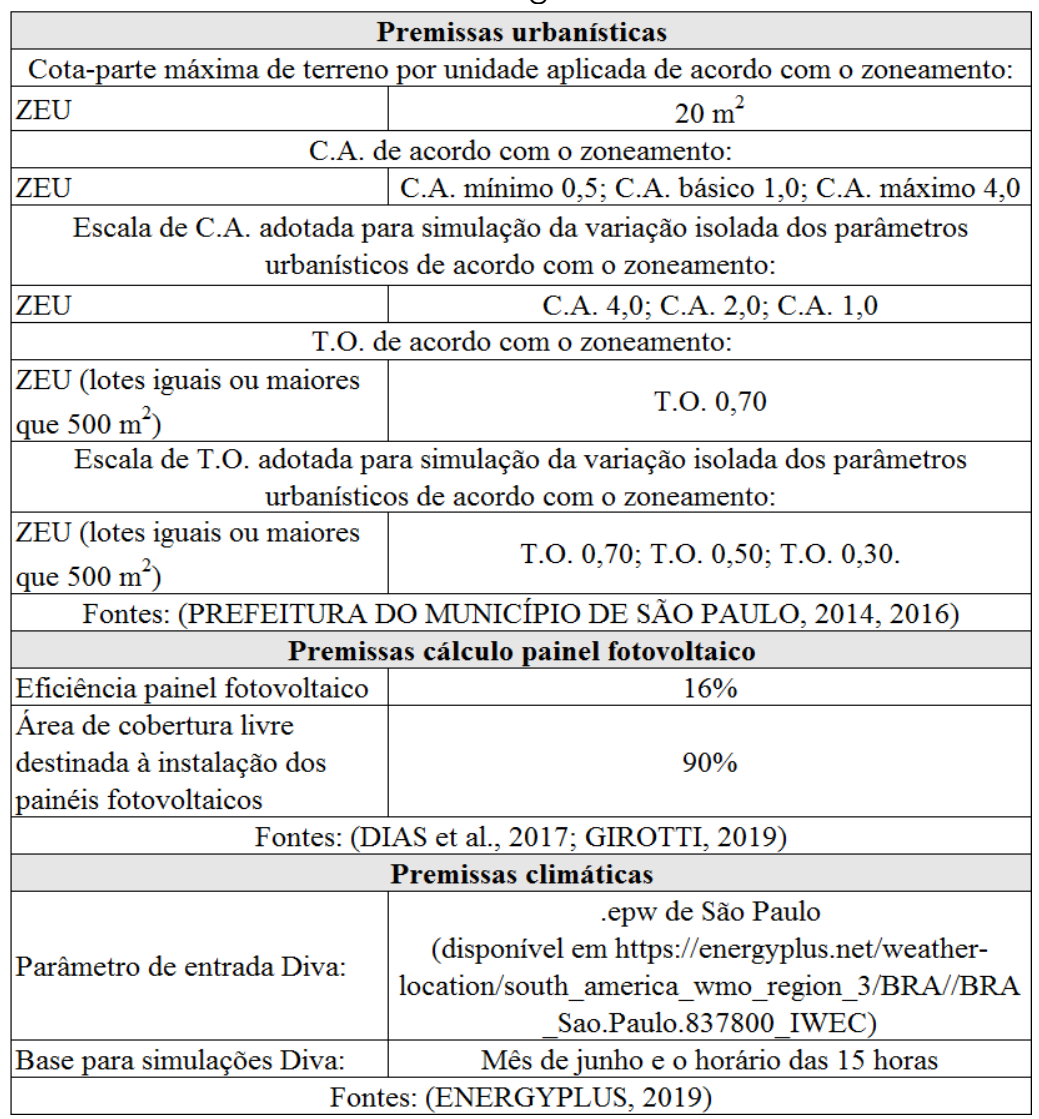

Fonte: Autoras, a partir de Dias et al. (2017) EnergyPlus (2019), Girotti (2019) e Prefeitura Município de São Paulo $(2014,2016)$

A visão geral das etapas apresentadas do algoritmo Grasshopper® é esquematizada pelo fluxograma da figura 2.

Figura 2 - Fluxograma das etapas desenvolvidas no algoritmo Grasshopper®

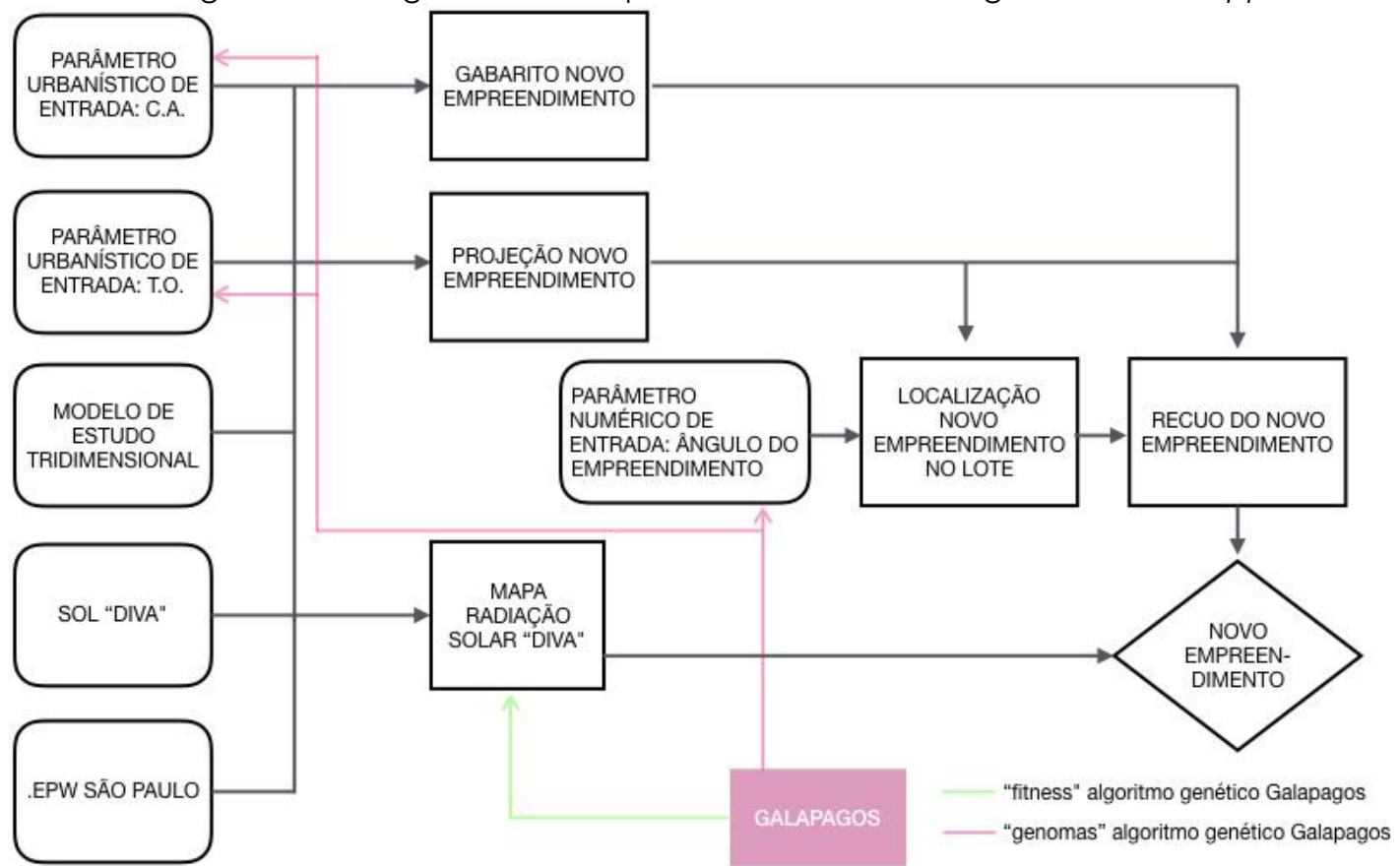

Fonte: As autoras 


\subsection{Apresentação da área de estudo de caso}

A figura 3 indica a localização do perímetro de estudo e seu parcelamento, uso e ocupação do solo de acordo com a Lei de Parcelamento, Uso e Ocupação do Solo do Município de São Paulo (PREFEITURA DO MUNICÍPIO DE SÃO PAULO, 2016).

Figura 3 -Parcelamento, uso e ocupação do solo no perímetro de estudo

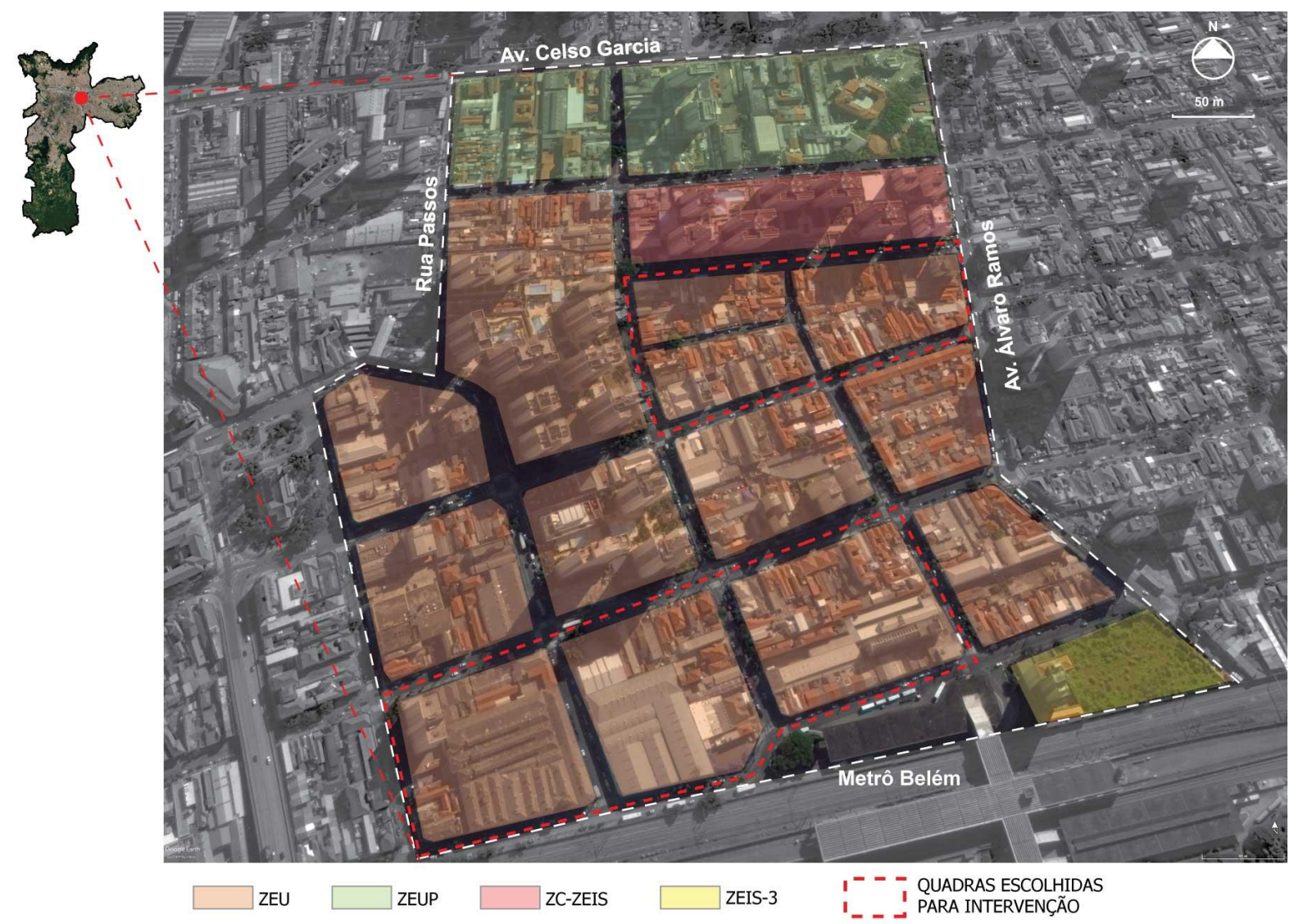

Fonte: Autoras, a partir da Lei de Parcelamento, Uso e Ocupação do Solo do Município de São Paulo (PREFEITURA DO MUNICÍPIO DE SÃO PAULO, 2016).

O uso e a ocupação do solo da área de estudo, predominantemente residencial, mas ainda com a presença de diversos galpões, no cenário dos incentivos construtivos previstos pelo Plano Diretor Estratégico para os EETU, torna o perímetro de estudo um local pertinente à realização da avaliação morfológica também no que se refere ao aproveitamento da energia fotovoltaica, conforme indica a figura 4, que ilustra o uso e a ocupação do solo da área de estudo e as quadras escolhidas para aplicação da metodologia. 
Figura 4 - Área de estudo: uso e ocupação do solo e lotes selecionados para com potencial de transformação do uso e ocupação do solo em médio prazo

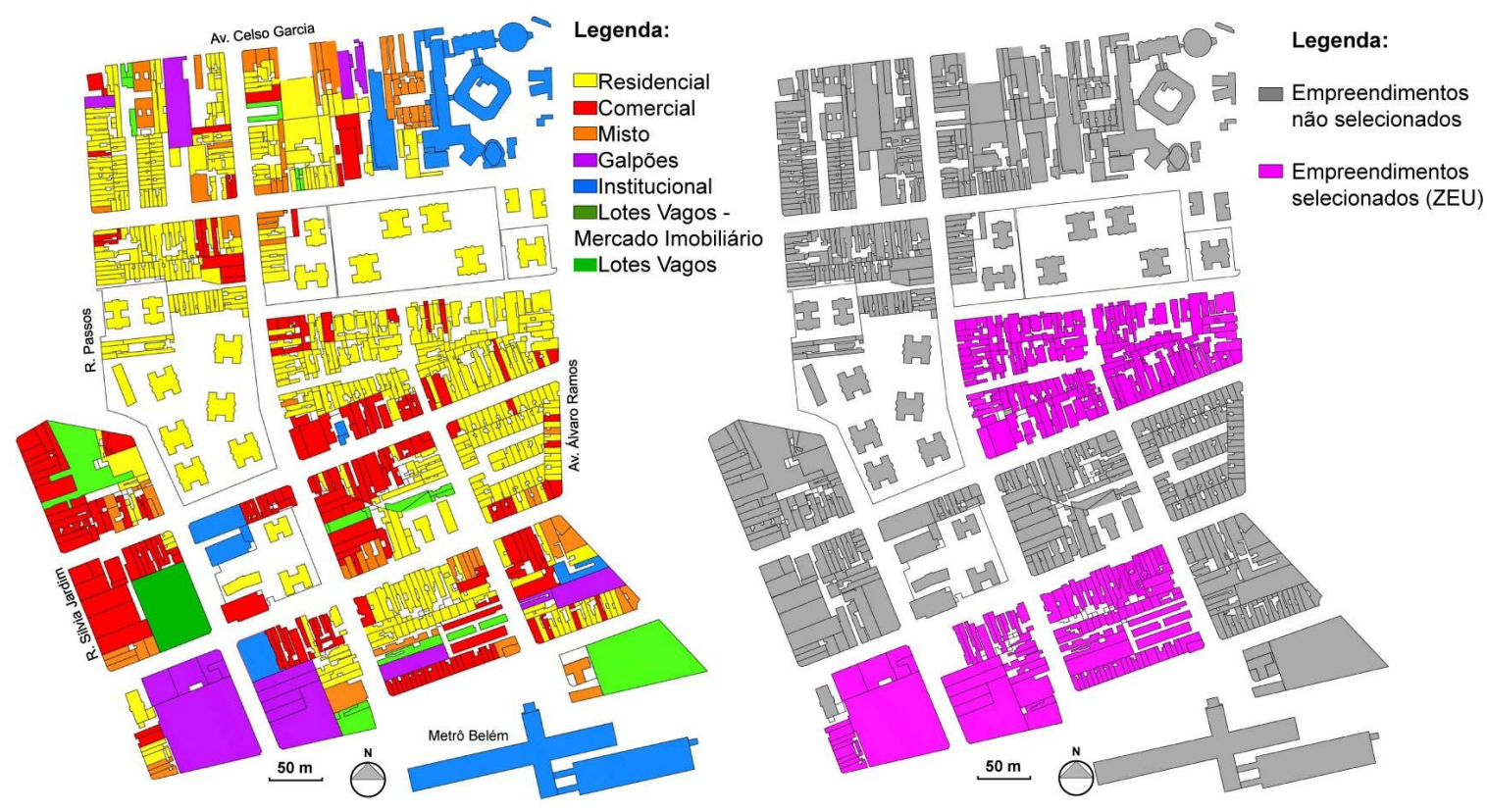

Fonte: As autoras

Foi realizado um levantamento acerca dos lotes com potencial de transformação na área de estudo, sendo definidos lotes concentrados, atualmente ocupados por residências e comércios antigos com baixa densidade construída e baixo gabarito de altura, com potencial de transformação do uso e ocupação do solo em médio prazo, para modelagem, simulação e análise do potencial de geração fotovoltaica.

A simulação da evolução morfológica da área em estudo considerou a intervenção em vinte e dois lotes, conforme ilustra a figura 5 , utilizando as ferramentas anteriormente descritas. As simulações das variações isolada (Grasshopper®) e simultânea (Grasshopper ${ }^{\circledR}+$ Galapagos) dos parâmetros urbanísticos foram baseadas em uma escala definida, indicado pelo quadro 1, considerando o zoneamento de cada lote de estudo.

Figura 5 - Lotes analisados na área de estudo: bairro do Belenzinho em São Paulo

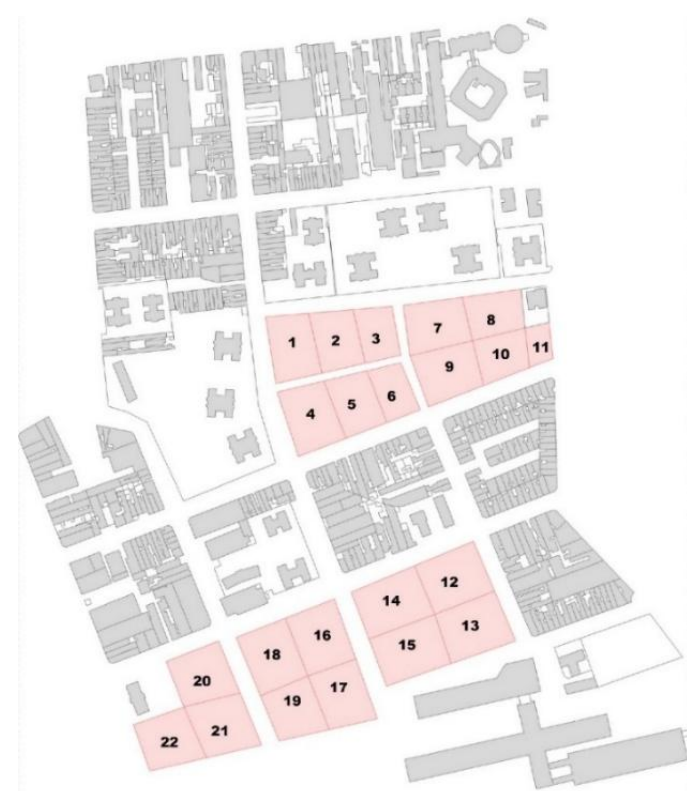

Fonte: As autoras 


\section{RESULTADOS OBTIDOS}

Ao comparar as simulações das variações dos parâmetros isolados, constatou-se que os resultados concorrem quando analisados por radiação solar incidente por metro quadrado e por radiação solar incidente por área total de cobertura. Quando a análise é realizada por radiação solar incidente por $\mathrm{m}^{2}$, boa parte dos melhores resultados se refere a taxas de ocupação menores, já que, dessa forma, os volumes construídos possuem gabarito de altura mais elevado e, portanto, estão mais expostos à radiação solar incidente por metro quadrado.

Porém, quando a análise é realizada considerando-se a radiação solar incidente por área total de cobertura, todos os resultados satisfatórios encontram-se nas variações que empregam maiores T.O. e C.A., segundo os valores permitidos pela legislação vigente no município de São Paulo, conforme ilustra a figura 6.

Figura 6 - Gráfico comparativo de incremento das melhores respostas por variação individual de parâmetros urbanísticos dos ganhos de radiação solar incidente por metro quadrado e por radiação solar incidente total, nos lotes considerados

\begin{tabular}{llllllllllllllllllllll}
4 & 4 & 4 & 4 & 4 & 4 & 4 & 4 & 4 & 4 & 4 & 4 & 4 & 4 & 4 & 4 & 4 & 4 & 4 & 4 & 4 & 4 \\
\hline
\end{tabular}

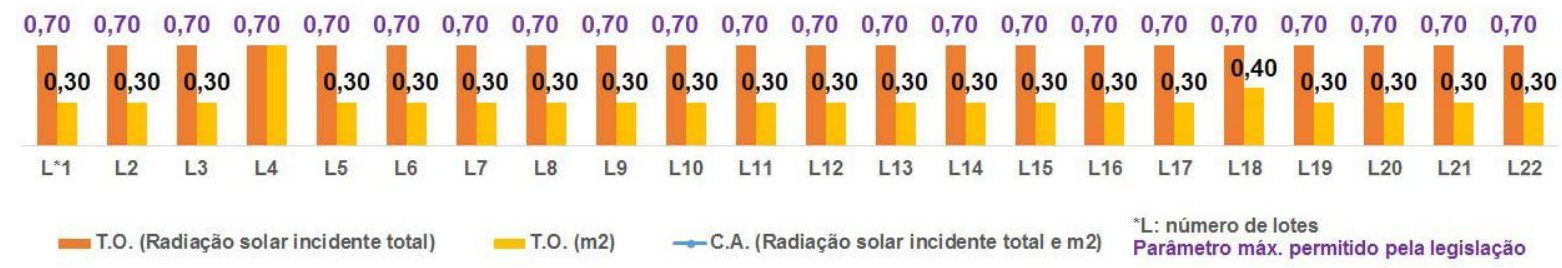

Fonte: As autoras

Os resultados obtidos na aplicação do algoritmo genético Galapagos não são condizentes com os resultados obtidos a partir da variação isolada dos parâmetros urbanísticos, pois quando o parâmetro urbanístico é variado de forma isolada, apenas o nível da radiação solar da cobertura do empreendimento analisado é considerado, sem considerar seus impactos no acesso à radiação solar do entorno. Já na simulação do Galapagos, o algoritmo genético busca uma melhor combinação entre os parâmetros analisados, de forma que encontre o melhor nível de radiação solar incidente na cobertura do empreendimento, mas mantendo níveis adequados na vizinhança. As simulações no Galapagos que encontraram os melhores resultados em relação ao nível de radiação solar referem-se às taxas de ocupação de 0,40 e 0,50 combinada com densidades construídas máximas ou intermediárias, conforme ilustra a figura 7.

Figura 7 - Gráfico comparativo de incremento das melhores respostas por variação combinada de parâmetros urbanísticos (Galapagos) dos ganhos de radiação solar incidente por radiação solar incidente total, nos lotes considerados

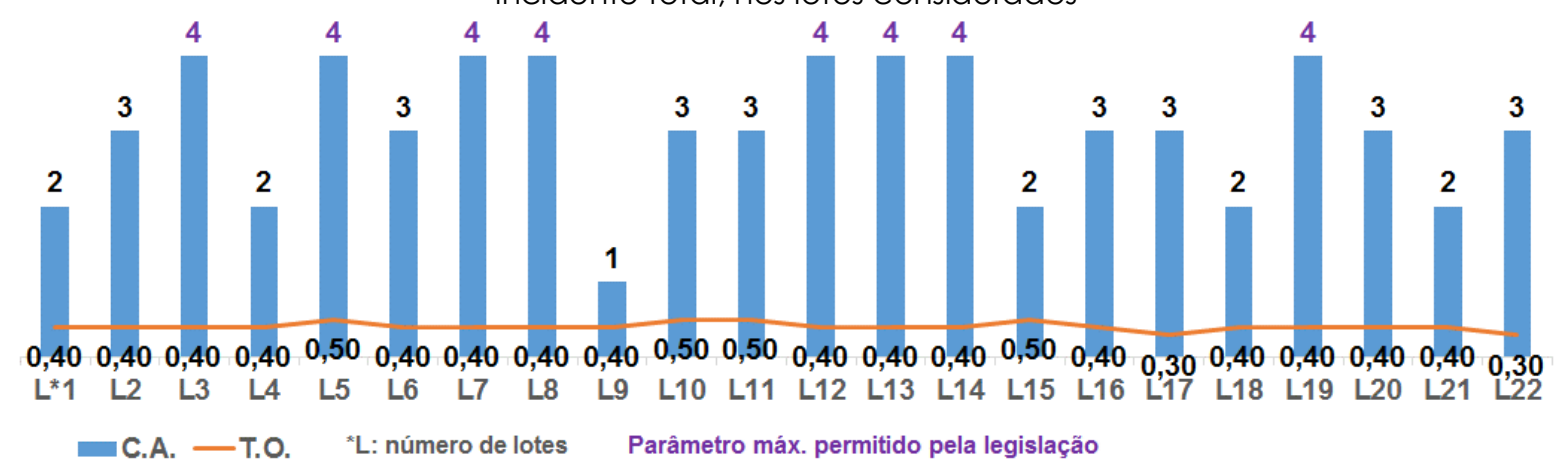

Fonte: As autoras

A figura 7 indica que, quando a legislação permite C.A. máximo de 4, o C.A. máximo 
representa $36 \%$ dos melhores resultados, contra $36 \%$ representados pelo valor intermediário de C.A. 3, 23\% representados pelo valor de C.A. 2 e $5 \%$ representados pelo valor C.A. 1.

Em relação à T.O., a figura 7 indica que o valor de 0,40 de T.O. representa $73 \%$ dos melhores resultados, contra $18 \%$ atribuído a valor de 0,50 de T.O e $9 \%$ atribuído a valor de 0,30. 0 resultado mostra que quando os lotes com intervenção morfológica estão concentrados, valores de T.O. menores possuem melhores respostas quando o objetivo é maximizar o nível de radiação solar na cobertura dos empreendimentos.

A comparação da morfologia urbana atual frente às propostas morfológicas, está ilustrada pela imagem tridimensional 8.

Figura 8 - Comparativo tridimensional entre os cenários morfológicos desenvolvidos no estudo

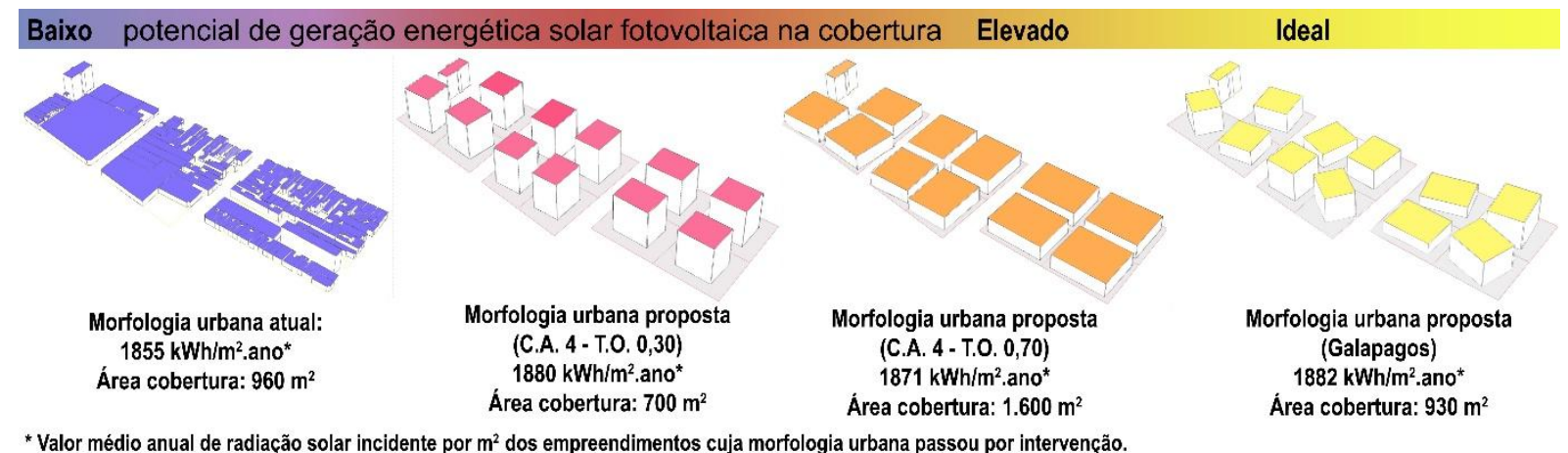

Fonte: As autoras

\section{CONCLUSÃO}

A análise dos resultados das simulações vai ao encontro da pesquisa dos autores Martins et al., (2016) e Martins, Adolpe e Bastos (2014) que indicam que quanto maior o número de pavimentos, maior será a radiação solar recebida pela edificação e que a distância entre as edificações é benéfica à maximização dos níveis de radiação solar incidente nas respectivas coberturas, por meio da adequação da T.O.

Dessa forma, a ferramenta Rhinoceros $₫$ associada aos plug-ins Grasshopper $₫$, Diva $®$ e ao algoritmo genético Galapagos, se mostraram adequadas para a simulação de diferentes valores para os parâmetros urbanísticos frente ao potencial de geração fotovoltaico. 0 Galapagos, em especial, é uma ferramenta que proporciona otimizar a morfologia urbana, por considerar não apenas ao empreendimento analisado, mas também o seu entorno, o que justifica a variação do gabarito de altura nos melhores resultados.

\section{AGRADECIMENTOS}

À Coordenação de Aperfeiçoamento de Pessoal de Nível Superior - Brasil (CAPES) - Código de Financiamento 001.

\section{REFERÊNCIAS}

ABBUD, O. A.; TANCREDI, M. Transformações Recentes da Matriz Brasileira de Geração de Energia Elétrica - Causas e Impactos Principais. Centro de Estudos da Consultoria do Senado. Brasil, p. 64, 2010.

ANTON, I.; TǍNASE, D. Informed Geometries. Parametric Modelling and Energy Analysis in Early Stages of Design. Energy Procedia, v. 85, p. 9-16, 2016.

BRASIL. Resolução Normativa no 482 de 2012 da ANEEL. ANEEL, p. 9, 2012.

BRASIL. Resolução Normativa no 687 de 2015 da ANEEL. ANEEL, p. 24, 2015. 
DIAS, C. L. DE A. et al. Performance estimation of photovoltaic technologies in Brazil.

Renewable Energy, v. 114, p. 367-375, 2017.

ENERGYPLUS. Weather Data by Location - Download - Sao Paulo 837800 (IWEC). Disponível em: <https://energyplus.net/weather-

location/south_america_wmo_region_3/BRA//BRA_Sao.Paulo.837800_IWEC>. Acesso em: 11 jun. 2019.

FEARNSIDE, P. M. Social Impacts of Brazil 's Tucuruí Dam. Environmental Management, v. 24, n. 4, p. 483-495, 1999.

FEARNSIDE, P. M. Dams in the Amazon: Belo Monte and Brazil's hydroelectric development of the Xingu River Basin. Environmental Management, v. 38, n. 1, p. 16-27, 2006.

FUTCHER, J. A.; MILLS, G. The role of urban form as an energy management parameter. Energy Policy, v. 53, p. 218-228, fev. 2013.

GAVIRIA, L. R.; PEREIRA, F. O. R.; MIZGIER, M. O. Influência da configuração urbana na geração fotovoltaica com sistemas integrados às fachadas. Ambiente Construído, v. 13, n. 4, p. 07-23, dez. 2013.

GIROTTI, Carolina. Modelagem dos parâmetros da forma urbana para a maximização de geração de energia solar fotovoltaica no ambiente urbano em adensamento e verticalização: estudo de caso do Belenzinho, em São Paulo. 2019. Dissertação (Mestrado em Engenharia de Construção Civil e Urbana) - Escola Politécnica, Universidade de São Paulo, São Paulo, 2019. doi:10.11606/D.3.2019.tde-27052019-132856.

MARINS, K. R. DE C. C.; ROMÉRO, M. DE A. Urban and Energy Assessment from a Systemic Approach of Urban Morphology, Urban Mobility, and Buildings: Case Study of Agua Branca in Sao Paulo. Journal of Urban Planning and Development, v. 139, n. 4, p. 280-291, dez. 2013.

MARTINO, J. A. DE; CELANI, G. Sistema Generativo Evolutivo como Método no Processo Criativo. Proceedings of the XVIII Conference of the Iberoamerican Society of Digital Graphics - SIGraDi: Design in Freedom, n. November, p. 380-383, 2014.

MARTINS, T. A. DE L. et al. Sensitivity analysis of urban morphology factors regarding solar energy potential of buildings in a Brazilian tropical context. Solar Energy, v. 137, p. 11-24, 2016.

MARTINS, T. A. DE L.; ADOLPE, L.; BASTOS, L. E. G. From solar constraints to urban design opportunities: Optimization of built form typologies in a Brazilian tropical city. Energy and Buildings, v. 76, p. 43-56, 2014.

PREFEITURA DO MUNICÍPIO DE SÃO PAULO. Plano Diretor Estratégico do Município de São Paulo.Brasil, 2014.

PREFEITURA DO MUNICÍPIO DE SÃO PAULO. Lei de parcelamento, uso e ocupação do soloBrasil, 2016.

ROBERT MCNEEL \& ASSOCIATES. Rhinoceros: versão 5.0, $2012 a$.

ROBERT MCNEEL \& ASSOCIATES. Grasshopper: versão 5.0, 2012b.

ROBINSON, D.; STONE, A. Irradiation modelling made simple: the cumulative sky approach and its applications. The $\mathbf{2 1}$ st Conference on Passive and Low Energy Architecture, Eindhoven, The Netherlands, n. September, p. 1-5, 2004. 
SANTOS, I. G. DOS; AUER, T.; SOUZA, R. V. G. DE. Optimized indoor daylight for tropical dense urban environments. Ambiente Construído, v. 17, n. 3, p. 87-102, 2017.

SARRALDE, J. J. et al. Solar energy and urban morphology: Scenarios for increasing the renewable energy potential of neighbourhoods in London. Renewable Energy, v. 73, p. 10-17, jan. 2015.

SOLEMMA. Diva: versão 4.0, 2016. 